\title{
THE INTEGRAL MISSION
}

\author{
W. HERMSEN ${ }^{1}$ AND C. WINKLER ${ }^{2}$ \\ ${ }^{1}$ Space Research Organization Netherlands \\ Sorbonnelaan 2, NL-3584 CA Utrecht, The Netherlands \\ ${ }^{2}$ ESA/ESTEC, Space Science Department, Astrophysics Div. \\ NL-2200 AG Noordwijk, The Netherlands
}

On behalf of the INTEGRAL Science Working Team

\section{Introduction}

The International Gamma-Ray Astrophysics Laboratory (INTEGRAL) is dedicated to the fine spectroscopy $(\triangle \mathrm{E}: 2 \mathrm{keV} F W H M @ 1 \mathrm{MeV})$ and fine imaging (angular resolution: 12' FWHM) of celestial gamma-ray sources in the energy range $15 \mathrm{keV}$ to $10 \mathrm{MeV}$. INTEGRAL was selected in 1993 as the next ESA medium-size scientific mission (M2) to be launched in 2001. ESA has the overall spacecraft and mission resposibilities, Russia will provide a PROTON launcher and launch facilities, and NASA will provide ground station support through the Deep Space Network. The scientific payload complement and the INTEGRAL Science Data Centre (ISDC) will be provided by large collaborations led by Principal Investigators (PI).

The INTEGRAL observatory will provide to the science community at large an unprecedented combination of imaging and spectroscopy over a wide range of $\mathrm{X}$-ray and gamma-ray energies including optical monitoring.

\section{Scientific Objections}

INTEGRAL is a $15 \mathrm{keV}$ - $10 \mathrm{MeV}$ gamma-ray mission with concurrent source monitoring at $\mathrm{X}$-rays $(3-35 \mathrm{keV})$ and in the optical range (550 - 850 nm). All instruments - co-aligned with large FOV's - cover simultaneously a very broad energy range when observing high energy sources.

The scientific goals of INTEGRAL will be attained by fine spectroscopy $(\Delta \mathrm{E}: 2 \mathrm{keV}$ FWHM @ $1 \mathrm{MeV}$ ) with fine imaging (angular resolution: 12' FWHM) and accurate positioning of celestial sources of gamma-ray emis- 
sion. Fine spectroscopy over the entire energy range will permit spectral features to be uniquely identified and line profiles to be determined for physical studies of the source region. The fine imaging capability of INTEGRAL within a large field of view will permit the accurate location and hence identification of the gamma-ray emitting objects with counterparts at other wavelengths, enable extended regions to be distinguished from point sources and provide considerable serendipitous science. In summary, the scientific topics to be addressed include: - Compact Objects (White Dwarfs, Neutron Stars, Black Hole Candidates) • Stellar Nucleosynthesis (Hydrostatic Processes, Supernovae, Novae) • High Energy Transients • Galactic Structure (Cloud Complex Regions, Mapping of continuum and line emission, ISM, CR distribution) • The Galactic Centre • Particle Processes and Acceleration - Transrelativistic Pair Plasmas • Extragalactic Astronomy (Nearby Galaxies, Clusters, AGN, Cosmic Diffuse Background) - Identification of High Energy Sources • Unidentified Gamma-Ray Objects as a Class • PLUS: Unexpected Discoveries.

\section{Scientific Payload}

The INTEGRAL payload consists of two main gamma-ray instruments: Spectrometer SPI and Imager IBIS, and of two monitor instruments, the $\mathrm{X}$-ray Monitor JEM-X and the Optical Monitoring Camera OMC.

The design of the INTEGRAL instruments is largely driven by the scientific requirement to achieve - to the maximum extent possible - complementarity in fine spectroscopy and accurate imaging. Therefore, the main gamma-ray instruments, SPI and IBIS, are differently optimised in order to complement each other and to achieve overall excellent performance. This optimisation also takes recent observations at high energies into account, which show that - in general - line emissions do occur on a wide range of angular and spectral extent: That is, broad lines seem preferably to be emitted from point-like sources and narrower lines from extended sources. The INTEGRAL continuum and line sensitivities are increased by an order of magnitude or more compared to succesful earlier instruments like SIGMA (continuum) and OSSE and COMPTEL (lines). The two monitor instruments (JEM-X and OMC) will provide complementary observations at X-ray and optical energy bands. A short overview of the INTEGRAL payload follows:

- The Spectrometer SPI will perform spectral analysis of gamma-ray point sources and extended regions with an energy resolution of $2 \mathrm{keV}$ (FWHM) at $1 \mathrm{MeV}$. This will be accomplished using an array of 19 hexagonal high purity Germanium detectors cooled by active cooling to an operating temperature of $85 \mathrm{~K}$. The total detection area is $500 \mathrm{~cm}^{2}$. A hexagonal 
coded aperture mask is located $1.7 \mathrm{~m}$ above the detection plane in order to image large regions of the sky (fully coded field of view $=16^{\circ}$ ) with an angular resolution of $2^{\circ}$.

In order to reduce background radiation, the detector assembly is shielded by an active BGO veto system which extends around the bottom and side of the detector almost completely up to the coded mask.

- The Imager IBIS provides powerful diagnostic capabilities of fine imaging (12 arcmin FWHM), source identification and spectral sensitivity to both continuum and broad lines over a broad (15 keV - $10 \mathrm{MeV})$ energy range. The energy resolution is $<7 \mathrm{keV} @ 0.1 \mathrm{MeV}$ and $60 \mathrm{keV} @ 1 \mathrm{MeV}$. A tungsten coded aperture mask (located at $3.2 \mathrm{~m}$ above the detection plane) is optimised for high angular resolution imaging. Sources $(>10 \sigma)$ can be located to $<60^{\prime \prime}$. As diffraction is negligible at gamma-ray wavelengths, the angular resolution obtainable with a coded mask telescope is limited by the spatial resolution of the detector array. The IBIS design takes advantage of this by utilising a detector with a large number of spatially resolved pixels, implemented as physically distinct elements.

The detector uses two planes, a front layer $\left(2600 \mathrm{~cm}^{2}\right)$ of CdTe pixels, each $(4 \times 4 \times 2) \mathrm{mm}(\mathrm{wxdxh})$, and a second one $\left(3100 \mathrm{~cm}^{2}\right)$ of CsI pixels, each $(9 \times 9 \times 30) \mathrm{mm}$. The aperture is restricted by a passive tungsten shield. The detector array is shielded in all other directions by a BGO scintillator veto system.

- The Joint European $X$-Ray Monitor JEM-X supplements the main INTEGRAL instruments and plays a crucial role in the detection and identification of the gamma-ray sources and in the analysis and scientific interpretation of INTEGRAL gamma-ray data. JEM-X will make observations simultaneously with the main gamma-ray instruments and provides images with $3^{\prime}$ angular resolution in the $3-35 \mathrm{keV}$ prime energy band.

The baseline photon detection system consists of two identical high pressure imaging microstrip gas chambers (Xenon at 5 bar) each viewing the sky through a coded aperture mask ( $4.8^{\circ}$ fully coded FOV), located at a distance of $3.2 \mathrm{~m}$ above the detection plane. The total detection area is $1000 \mathrm{~cm}^{2}$.

- The Optical Monitoring Camera OMC consists of a passively cooled CCD in the focal plane of a $50 \mathrm{~mm}$ lens. The CCD (1024 x 2048 pixels) uses one section (1024 x 1024 pixels) for imaging, the other one for frame transfer before readout. The FOV is $5^{\circ} \times 5^{\circ}$ with a pixel size of 17.6". The OMC will observe the optical emission from the prime targets of the INTEGRAL main gamma-ray instruments with the support of the $\mathrm{X}$-Ray Monitor JEM-X. OMC offers the first opportunity to make long observations in the optical band simultaneously with those at X-rays and gamma-rays. Variability patterns ranging from 10's of seconds, hours, up to 
months and years will be monitored. The limiting magnitude will be $19.2^{m_{v}}$ $\left(3 \sigma, 10^{3} \mathrm{~s}\right)$, which corresponds to $\sim 40$ photons $\mathrm{cm}^{-2} \mathrm{~s}^{-1} \mathrm{keV}^{-1}$ (@ $2.2 \mathrm{eV}$ ) in the $\mathrm{V}$-band. Multi-wavelength observations are particularly important in high-energy astrophysics where variability is typically rapid. The wide band observing opportunity offered by INTEGRAL is of unique importance in providing for the first time simultaneous observations over seven orders of magnitude in photon energy for some of the most energetic objects.

\section{INTEGRAL Science Data Centre}

The ISDC, located in Versoix, Switzerland, will receive the complete raw science telemetry plus the relevant ancillary spacecraft data from the Mission Operation Centre in Darmstadt. Science data will be processed, taking into account the instrument characteristics, and raw data will be converted into physical units. Using incoming science and housekeeping information, the ISDC will routinely monitor the instrument science performance and conduct a quick-look science analysis. Most of the Targets of Opportunity (TOO) showing up during the lifetime of INTEGRAL will be detected at the ISDC during the routine scrutiny of the data. Final data products obtained by standard analysis tools will be distributed to the observer and archived for later use by the science community. Facilities will be provided to support the science community in the analysis of INTEGRAL data.

\section{Observing Programme}

INTEGRAL will be an observatory-type mission with a nominal lifetime of 2 years, an extension up to 5 years is technically possible. The parameters for the baseline orbit (PROTON launcher) are: period 48 hours, inclination $51.6^{\circ}$, perigee height $46000 \mathrm{~km}$, apogee height $75000 \mathrm{~km}$. In fact, $100 \%$ of time in orbit can be used for observations. Most of the observing time (65\% during year $1,70 \%$ (year 2 ), $75 \%$ (year $3+$ )) will be awarded to the scientific community at large as the General Programme. Proposals, following a standard AO process, will be selected on their scientific merit only by a single Time Allocation Committee. The remaining fraction of the observing time (i.e. $35 \%$ (year 1), $30 \%$ (year 2), $25 \%$ (year $3+$ )) will be reserved, as guaranteed time, for, mainly: (i) the institutes (PI collaborations) which have developed and delivered the instruments and the ISDC (guaranteed PI time), and (ii) for Russia and NASA for their contributions to the programme (PROTON launcher and Deep Space Network ground stations).

For more detailed information on INTEGRAL please consult the INTEGRAL pages on the WWW:

http://astro.estec.esa.nl/SA-general/Projects/Integral/integral.html 